\title{
PROBLEMAS PERCEBIDOS COMO PRIORITÁRIOS PELOS PACIENTES EM DIÁLISE PERITONIAL
}

\author{
Djair Daniel Nakamae* \\ Francisca Muniz** \\ Helma Hermann *** \\ Lorita Pagliuca $* * *$
}

NAKAMAE, D. D.; MUNIZ, F.; HERMANN, H.; PAGLIUCA, L. Problemas percebidos como prioritários pelos pacientes em diálise peritonial. Rev. Esc. Enf. USP, São Paulo, 13(2):115-125, 1979.

As autoras procuraram identificar os problemas de enfermagem que afetam prioritariamente os pacientes em diálise peritoneal. Objetivam também estabelecer bases para, num próximo trabalho, determinar tratamento apoiado $\mathrm{em}$ dados mais seguros. $O$ estudo mostrou que em grande número dos pacientes entrevistados houve concordância entre os problemas sentidos e a expectativa de atendimento pela enfermagem.

\section{INTRODUÇÃO}

O ensino da assistência ao paciente renal, na disciplina Enfermagem Médica, área de atuação das autoras, tem como ponto de partida a minuciosa identificação dos problemas de enfermagem realizada pelos alunos sob orientação da professora.

0 doente representa um papel passivo, pouco se conhecendo acerca dos problemas a que dá maior importância e de suas expectativas quanto à assistência a que tem direito.

Isso é particularmente verdadeiro nas salas de diálise peritoneal. Observações empíricas e freqüentes aí realizadas vêm causando preocupação entre as docentes, pelo fato de que esses pacientes nem sempre se mostram totalmente em conforto diante da assistência definida como prioritária pelos alunos ou pelas enfermeiras de campo.

É oportuno indicar que, hoje, há um consenso quase geral em torno da tese de que as necessidades básicas do homem precisam ser constantemente atendidas, a fim de manter o seu completo bem-estar físico, psíquico e social ${ }^{5}$. Quando adocce, o ser humano é afetado como um todo e suas necessidades passam a se manifestar como problema.

Sabe-se, também, que o indivíduo hospitalizado passa a assumir e a desempenhar um papel específico; dai o fato de o paciente portador de afecção renal e em tratamento dialítico poder apresentar uma série de problemas advindos da área patológica, da área global, da condição de doente hospitalizado e do tratamento específico, que é a diálise peritoneal.

* Professor Assistente da disciplina Enfermagem Médico-Cirúrgica da EEUSP. Mestre em Enfermagem.

** Diretor da Faculdade de Enfermagem São José.

*** Professor Assistente do Departamento de Enfermagem da Escola Paulista de Medicina. 
Na literatura sobre o assunto, pouco se encontrou. A grande maioria dos trabalhos se detém exclusivamente na técnica de terapia dialítica, sem se preocupar com a percepção de problemas sentidos pelo paciente. Isso motivou a realização desta pesquisa, a qual se propõe a identificar com pormenores os problemas de enfermagem que afetam prioritariamente tais pacientes, com o objetivos de se conseguir um ponto de partida para novos trabalhos, que determinem tratamento de enfermagem em bases mais seguras. Tais questões parecem úteis não apenas à enfermeira que presta cuidados diretos na sala de diálise, mas, também, e principalmente, à docente de enfermagem que necessita orientar teórica e praticamente os alunos no atendimento a esses doentes.

A partir de 1953, encontram-se na literatura norte americana grande número de estudos sobre a necessidade do levantamento de problemas de enfermagem para, a seguir, ser determinado com maior segurança o tratamento específico de enfermagem.

DICHTER ${ }^{4}$ realizou estudo tendo como tema o "relacionamento paciente-hospital"; chegou à conclusão de que o paciente hospitalizado sofre modificações profundas em sua personalidade, tornando-se emocionalmente uma criança. Refere ser a necessidade emocional o "primeiro fator não médico que precisa ser atendido". Conclui que essas características emocionais devem ser tomadas em consideração na assistência ao paciente, indicando ainda que a expressão da insegurança básica deste é manifestada por queixas a respeito de coisas que ele se sente capaz de avaliar, tais como o pouco contato com as enfermeiras, o não atendimento de algumas solicitações, etc.

ABDELLAH \& LEVINE ${ }^{2}$ relatam conclusões semelhantes às de DICHTER ${ }^{4}$ em relação ao doente hospitalizado. Dizem que a "situação hospitalar é de tensão e as necessidades percebidas passam a ser de grande importância para o doente". 0 não atendimento destas agrava o quadro da doença e que, quando satisfeitas, possibilitam ao paciente reagir ao tratamento mais facilmente.

ABDELLAH ${ }^{1}$, num estudo significativo, diz que a assistência eficiente é aquela $\mathrm{em}$ que o paciente faz a avaliação dos cuidados recebidos em relação às necessidades por ele percebidas. Afirma, ainda, que "talvez estejam satisfazendo às necessidades dos hospitais, dos médicos e das enfermeiras, mas não as dos pacientes".

No Brasil, KAMIYAMA ${ }^{6}$ realizou trabalho de relevante importância, que inspirou e serviu de base para o presente estudo. Interrogou 113 pacientes adultos e hospitalizados em Clínicas Médicas e Cirúrgicas, em relação à sua percepção quanto à prioridade de seus problemas. Das conclusões obtidas pela autora, mencionam-se estas: "os problemas considerados prioritários pelos pacientes são relativos ao medo ao desconhecido, à família, à sintomatologia clínica e ao estado geral; os problemas em relação às expectativas de atendimento pelas enfermeiras, sentidos como prioritários, são relativos ao medo do desconhecido, à sintomatologia clínica e estado geral e à alimentação". Adverte que, "para iniciar a coleta de dados para o diagnóstico de enfermagem, parece ser indicado o levantamento dos problemas sentidos pelos pacientes, através de um formulário com perguntas de respostas abertas". 
Dentro dos limites propostos pelo estudo pretende-se:

1. identificar os problemas percebidos como prioritários pelos pacientes em diálise peritoneal;

2. estabelecer bases para, num futuro trabalho, determinar que tratamentos de enfermagem são mais eficientes e mais adequados para os problemas identificados.

\section{Definição de termos}

Para uso operacional serão utilizadas algumas definições elaboradas por vários autores e usadas em trabalhos anteriores.

Problemas sentidos pelos pacientes - KAMIYAMA ${ }^{6}$ define-os como sendo "aqueles que os pacientes conscientizam ou percebem como problemas e conseguem verbalizar".

Problema de enfermagem - Segundo ABDELLAH 1, é "uma situação enfrentada pelo paciente ou família e para cuja solução o enfermeiro pode ajudar, através do desempenho de suas funções profissionais".

Necessidades básicas humanas - Para KAMIYAMA ${ }^{6}$ são "condições que precisam ser atendidas para que o indivíduo conserve a vida e o bem-estar, condições essas resultantes de momentos de desequilíbrio na constância da dinâmica dos fenômenos vitais".

\section{Amostra estudada}

\section{METODOLOGIA}

0 presente estudo baseou-se em dados de uma amostra de 50 pacientes provenientes das Unidades de Diálise de cinco hospitais gerais da cidade de São Paulo, questionados no decorrer de 1976.

Seleção dos pacientes

A amostra em estudo obedeceu aos seguintes critérios básicos:

a) pacientes adultos, de 20 e 60 anos de idade, pois, segundo as observações de ABDELLAH \& LEVINE ${ }^{3}$, existem diferenças marcantes entre o adolescente e o indivíduo de mais de 60 anos na maneira de sentir e de avaliar as situações;

b) brasileiros, residentes no Brasil, excluindo-se os naturalizados. KAMIYAMA ${ }^{6}$ usou esse critério a fim de tentar afastar influências de diferenças culturais na percepção e expressão dos problemas;

c) pacientes em diálise peritonial não considerado o número de internações anteriores, porém, com um mínimo de 12 horas de tratamento, para que fosse possivel a verificação dos problemas advindos do tratamento dialítico;

d) pacientes conscientes e lúcidos, capazes de manter uma entrevista. 
Foi efetuada apenas uma visita para cada paciente, com o intuito de questionálos sobre seus problemas prioritários.

\section{Caracteristicas da amostra estudada}

Do total de pacientes que compunham a amostra $29(58,0 \%)$ pertenciam ao sexo masculino e $21(42,0 \%)$ ao sexo feminino.

Quanto à idade, verificou-se que $16(32,0 \%)$ enquadravam-se na faixa de 20 a 30 anos; $16(32,0 \%)$, na de 31 a 40 anos; $11(22,0 \%)$, na de 41 a 50 anos e $7(14,0 \%)$, na de 51 a 60 anos de idade. pardos.

Em relação à cor, $41(82,0 \%)$ eram brancos, $7(14,0 \%)$ pretos e $2(4,0 \%)$

Sobre o estado civil, $32(64,0 \%)$ informaram ser casados e $17(34,0 \%)$, solteiros. Dos casados, 78,0\% informaram ter filhos.

Tendo em conta o total de informantes, constatou-se que $23(46,0 \%)$ eram provenientes do Estado de São Paulo; 8(16,0\%) de Minas Gerais; $8(16,0 \%)$ da Bahia; $3(6,0 \%)$ do Paraná; $2(4,0 \%)$ de Goiás; $2(4,0 \%)$ de Pernambuco e os 4 restantes de Alagoas, Ceará, Mato Grosso e Rio Grande do Sul.

\section{Instrumento}

Os dados foram coletados por meio de um formulário, em anexo, preenchido em entrevista e contendo três questões abertas, no qual se tomaram por base quesitos aplicados na pesquisa de KAMIYAMA ${ }^{6}$. Foi preliminarmente testado em cerca de oito pacientes escolhidos ao acaso em cinco hospitais da capital, no mês de maio de 1975, tendo sofrido algumas correções (com exclusão e adaptação de perguntas).

Os itens pesquisados podem ser agrupados em três categorias:

a) as "coisas" mais importantes que incomodam o paciente por estar em diálise peritoneal;

b) as "coisas" que fazem mais falta por estar acamado na sala de diálise;

c) o que acha que as enfermeiras devem fazer por ele.

\section{Aplicação do instrumento}

A aplicação dos formulários foi realizada obedecendo-se à técnica de entrevista; quatro pesquisadoras, devidamente treinadas, levantaram os dados nos cinco hospitais, no decorrer de 1976.

Antes do início da entrevista coletaram-se os dados dos pacientes, através da consulta à papeleta e ao prontuário médico de cada um deles, obedecendo-se, para seleção, aos critérios adotados no planejamento. Esses dados foram completados através de indagações à enfermeira e ao médico da Unidade.

Por ocasião da entrevista procurou-se motivar os pacientes; foi-lhes explicado o propósito do estudo, garantido o anonimato de suas respostas e pedida sua colaboração. De modo geral, as entrevistadoras foram bem recebidas pelos pacientes e ne- 
nhum deles se negou a cooperar. Por parte das enfermeiras responsáveis pelas Unidades houve pronta autorização para aplicação dos formulários e algumas até tomaram providências para apresentar a pesquisdora aos pacientes e facilitar seu acesso ao prontuário e à papeleta dos mesmos.

O tempo gasto para cada entrevista variou de dez a vinte e cinco minutos.

\section{ANÁLISE E DISCUSSÃO DOS DADOS}

Nesta seção pretende-se oferecer uma visão dos problemas percebidos como prioritários pelos pacientes em diálise peritoneal.

Os dados obtidos são analisados obedecendo-se à seguinte seqüência:

- os problemas percebidos pelos pacientes com maior intensidade;

- as expectativas dos pacientes quanto à assistência de enfermagem.

Este segundo item foi colocado com a finalidade de confirmar a veracidade do primeiro.

1. Problemas percebidos pelos pacientes com mais intensidade

$\mathrm{Na}$ análise deste item julgou-se necessário interrogar o paciente em relação às "coisas" mais importantes que o incomodam por estar em diálise peritoneal e às "coisas" que lhe fazem mais falta por estar acamado na sala de diálise. Nas duas questōes foram registradas a primeira e a segunda prioridade referidas pelo paciente. Pretendeu-se, assim, delimitar entre as "coisas" que INCOMODAM E LHE FAZEM FALTA, quais as mais importantes em ordem de valor.

a) As "coisas" mais importantes que o incomodam por estar em diálise peritoneal

$\mathrm{Na}$ tabela 1 podem ser apreciados os dados relativos a este item. As respostas que se referem ao tratamento dialítico propriamente dito mostram grande predom minância em relação às demais, abrangendo $80,0 \%$ das respostas em primeira prioridade e 16,0\% em segunda. Assim, a grande maioria dos pacientes amostrados $72,0 \%$ acusam como queixa principal a DOR ABDOMINAL SENTIDA DURANTE O TRATAMENTO DIALfTICO, enquanto que $16,0 \%$ a referem como queixa de segunda opção. Todavia, apenas $8,0 \%$ apontam como prioritária a DOR QUE RESULTA DA INSTALAÇÃO DO CATÉTER PERITONEAL.

Os problemas relativos ao DESCONFORTO DURANTE A DIÁLISE PERITONEAL foram apontados por $10,0 \%$ dos pacientes amostrados, como primeira queixa, e por $52,0 \%$ como segunda. Esses problemas, em ordem decrescente de freqüência, são os seguintes:

- desconforto durante a movimentação no leito;

- posição desconfortável no leito;

- o fato de permanecer acamado;

— o fato de ser acordado à noite para controles; 
- colheita de sangue duas vezes ao dia;

— sonda vesical e "intracath";

- tudo traz desconforto.

Com menor incidência, como se pode verificar na tabela 1 , estão os problemas relativos à ALIMENTAÇÃO E HIDRATAÇÃO, abrangendo 4,0\% e 2,0\%, respectivamente em primeira e segunda importância; referem-se à alimentação sem gosto, sensação de muita fome e sensação de sede.

As queixas relativas à SINTOMATOLOGIA CLINICA, com 2,0\% e 10,0\%, em primeira e segunda prioridade respectivamente, acusam os seguintes problemas: dor lombar, tonturas, insônia noturna, vômitos, fraqueza geral e constipação intestinal. Convém ressaltar que, na quase totalidade, os pacientes entrevistados eram portadores de Insuficiência Renal Crônica e esta sintomatologia é bem típica, aparecendo em freqüência bem baixa, o que era de se esperar, em pacientes parcialmente compensados pelo programa de diálise crônica.

A falta de liberdade que incomoda $2,0 \%$ e $6,0 \%$ dos paciantes pesquisados, em ordem decrescente de importância, diz respeito a ambiente fechado e pequeno, permanência longa na sala de diálise, impossibilidade de ir ao sanitário e dependência de outros para o seu cuidado pessoal.

TABELA 1

Problemas percebidos com mais intensidade pelos pacientes, segundo o que mais os incomoda por estarem em diálise peritoneal.

\begin{tabular}{|c|c|c|c|c|}
\hline \multirow[t]{2}{*}{ Prioridades } & \multicolumn{2}{|c|}{ Primeira } & \multicolumn{2}{|c|}{ Segunda } \\
\hline & $F$ & $\%$ & $\mathbf{F}$ & $\%$ \\
\hline Relativos a: & & & & \\
\hline dor abdominal proveniente de tratamento dialítico & 40 & 80,0 & 8 & 16,0 \\
\hline desconforto durante a diálise & 5 & 10,0 & 26 & 52,0 \\
\hline alimentação e hidratação & 2 & 4,0 & 1 & 2,0 \\
\hline sintomatologia clínica & 1 & 2,0 & 5 & 10,0 \\
\hline falta de liberdade & 1 & 2,0 & 3 & 6,0 \\
\hline sem problemas & 1 & 2,0 & 7 & 14,0 \\
\hline Total & 50 & 100,0 & 50 & 100,0 \\
\hline
\end{tabular}

Constata-se, assim, que a DOR e o DESCONFORTO, resultantes da diálise peritoneal constituem a grande queixa que predomina tanto em primeira como em segunda importância na escala de valores apontada pelos pacientes pesquisados. Ao mesmo tempo, o incômodo advindo da Alimentação, Hidratação, Sintomatologia clínica e Falta de liberdade constitui a minoria dos seus infortúnios durante o tratamento dialítico.

b) As "coisas" que lhe fazem mais falta por estar acamado na sala de diálise

Verifica-se na tabela 2 que os problemas relativos à família foram os mais indicados pelos pacientes dentre as "coisas" que lhe fazem mais falta. Houve uma 
incidência de $48,0 \%$ em primeira prioridade $28,0 \%$ em segunda. Nesse grupo de problemas estão referidos os relativos à saudade da família, preocupação com a situação econômica dos familiares (relativas ao emprego e a compromissos).

Em ordem decrescente de freqüência a segunda queixa mais referida pelos pacientes em estudo diz respeito à RECREAÇÃO E ATIVIDADE, alcançando 18,0\% em primeira importância e 12,0\% em segunda. Nesse grupo foi indicado como fazendo-lhes falta: o rádio, a televisão, a deambulação, o trabalho habitual, as revistas, o fumo e a clínica de origem.

A alimentação e hidratação oferecidas na sala de diálise constituiram queixa muito pequena quando os pacientes foram questionados sobre as "coisas" que os incomodavam durante a diálise (como se indicou na Tabela 1). Todavia, quando inquiridos sobre as "coisas" que lhes fazem mais falta na sala de diálise, a percentagem desse item subiu significativamente. Assim, 14,0\% indicaram esse item como queixa principal e 12,0\% como queixa de segunda importância. Nesse aspecto disseram sentir falta de sal e tempero nos alimentos, de chá quente, de feijão preto e água.

Constata-se ainda na Tabela 2 que a ELIMINAÇÃO, não referida pelos pacientes com queixa na Tabela 1 , aparece agora em $8,0 \%$ e $2,0 \%$ (primeira e segunda prioridades, respectivamente) dos mesmos que dizem sentir falta do sanitário para defecar.

Entre os CUIDADOS PESSOAIS, a falta do banho de chuveiro foi apontada (Tabela 2) como problema principal por apenas $4,0 \%$ dos amostrados. Contudo, como segunda prioridade subiu a sua importância para $26,0 \%$ dos pacientes.

\section{TABELA 2}

Problemas sentidos com mais intensidade pelos pacientes, segundo o que mais lhes faz falta por estarem acamados na sala de diálise.

\begin{tabular}{l|rrrr}
\hline & \multicolumn{2}{c}{ Primeira } & \multicolumn{2}{c}{ Segunda } \\
Problemas & $\mathrm{F}$ & $\%$ & $\mathrm{~F}$ & $\%$ \\
\hline \multirow{2}{*}{ Relativos a: } & & & & \\
família & 24 & 48,0 & 14 & 28,0 \\
recreação e atividade & 9 & 18,0 & 6 & 12,0 \\
alimentação e hidratação & 7 & 14,0 & 6 & 12,0 \\
eliminações & 4 & 8,0 & 1 & 2,0 \\
cuidados pessoais & 2 & 4,0 & 13 & 26,0 \\
não tem & 2 & 4,0 & 9 & 18,0 \\
sem resposta & 2 & 4,0 & 1 & 2,0 \\
\hline \multicolumn{1}{c|}{ Total } & 50 & 100,0 & 50 & 100,0 \\
\hline
\end{tabular}

Os dados da Tabela 2 mostram, portanto, que, como primeira prioridade, houve maior concentração da frequêencia nos grupos de problemas relativos a: familia $(48,0 \%)$, recreação e atividade $(18,0 \%)$ e alimentação e hidratação $(14,0 \%)$; enquanto que, na segunda prioridade, a maior incidência foi relativa a familia $(28,0 \%)$, cuidados pessoais $(26,0 \%)$, recreação e atividades $(12,0 \%)$, alimentação e hidratação $(12,0 \%)$. 
A Tabela 3 mostra os dados relativos à expectativa dos pacientes amostrados diante dos problemas apontados anteriormente.

Como se viu na Tabela 1 , o incômodo trazido pela dor e o desconforto resultantes do tratamento dialítico constituem a queixa predominante dos pacientes. É interessante observar que, com bastante coerência, as expectativas dos pacientes referem-se, na sua grande maioria, ao tratamento dialítico e clínico e ao conforto físico e emocional. $O$ tratamento dialítico e clínico é apontado por $36,0 \%$ (em primeira prioridade e $28,0 \%$ (em segunda prioridade). coglobando as seguintes expectativas de atendimento pela enfermagem: pontualidade nas trocas dos banhos dialiticos, não drenar o banho até o fim, atendimento mais rápido às solicitações, prontidão na medicação para dormir e para a dor, explicação do que vai ser feito durante a diálise.

0 conforto físico e emocional foi indicado por $32,0 \%$ em primeira prioridade e por $30,0 \%$ em segunda. Entre eles citam-se as seguintes expectativas: ajudar a aguentar o tratamento e a sair logo da sala de diálise, cuidar bem, ser mais humana no contato; não acordar à noite para controles; trocar a roupa com cuidado; não colher tanto sangue; permitir o banho de chuveiro; fazer massagem de conforto; deixar quieto no leito; deixar ver lá fora.

Entre os pacientes restantes encontrou-se que: 10,0\% e 8,0\% (primeira e segunda prioridades) têm expectativas relativas à recreação e atividade; $8,0 \%$ ( tanto em primeira quanto em segunda prioridade) citam a alimentação e hidratação ( melhorar o sabor dos alimentos, dar mais água, mais comida e chá quente); enquanto que apenas $2,0 \%$, em segunda prioridade, esperam que a enfermagem facilite a sua comunicação com os familiares (Tabela 3 ).

É importante destacar a freqüência relativamente alta de pacientes que referem não ter expectativa alguma em relação ao atendimento de enfermagem $(14,0 \% \mathrm{em}$ primeira prioridade e $24,0 \%$ em segunda). Seria interessante detectar, em trabalhos futuros, a que fator se prende esse fato: se à indiferença dos pacientes, segundo seu estado geral, se a fatores do meio hospitalar ou a outras quaisquer.

TABELA 3

Expectativas dos pacientes quanto à assistência de enfermagem.

\begin{tabular}{|c|c|c|c|c|}
\hline$\ldots \quad$ Prioridades & & & & \\
\hline Problemas & $\mathbf{F}$ & $\%$ & $F$ & 0 \\
\hline \multirow[t]{2}{*}{$\begin{array}{l}\text { Relativos a: } \\
\text { tratamento dialftico e clínico } \\
\text { conforto físico e emocional } \\
\text { recreação e atividade } \\
\text { alimentação e hidratação } \\
\text { famflia (ausência) } \\
\text { não tem expectativa }\end{array}$} & $\begin{array}{r}18 \\
16 \\
5 \\
4 \\
-7\end{array}$ & $\begin{array}{r}36,0 \\
32,0 \\
10,0 \\
8,0 \\
-\end{array}$ & $\begin{array}{r}14 \\
15 \\
4 \\
4 \\
1 \\
12\end{array}$ & $\begin{array}{r}28,0 \\
30,0 \\
8,0 \\
8,0 \\
2,0 \\
24,0\end{array}$ \\
\hline & 50 & 100,0 & 50 & 100,0 \\
\hline
\end{tabular}




\section{CONCLUSÕES}

Nesta seção do trabalho, apresentam-se os problemas percebidos como prioritários pelos pacientes em diálise peritoneal, mediante uma descrição sucinta dos mesmos, conforme o objetivo da presente pesquisa.

- Os pacientes em diálise peritoneal constituem um grupo típico, com problemas prioritários comuns ao grupo.

- Os problemas percebidos pelos pacientes como prioritários e que se apresentam com freqüência alta na primeira prioridade, com rara exceção, apresentam-se também em segunda opção.

- Os problemas percebidos com mais intensidade por estes pacientes durante o tratamento dialítico, em escala de prioridade, se referem a:

- dor e desconforto provenientes da diálise

- família

- recreação e atividade

- alimentação

- As expectativas dos pacientes relativas ao atendimento de enfermagem, citados em escala de prioridade, se referem a:

- dor e desconforto provenientes do tratamento

- recreação e atividade

- alimentação e hidratação

- Em grande número dos pacientes entrevistados houve concordância, pelo menos uma, entre os problemas sentidos e a expectativa de atendimento pela enfermagem. 
NAKAMAE, D. D.; MUNIZ, F.; HERMANN, H.; PAGLIUCA, L. Nursing problems affecting patients in peritoneal dialysis. Rev. Esc. Enf. USP, São Paulo, 13(2):115-125, 1979.

The authors try to identify the main nursing problems affecting patients in peritoneal dialysis. They intend, also, to find some stand points in order to determine, in a next survey, a treatment based on more accurate data. This study show that for a large number of the patients, there is a mutual relation between the problems perceived and the expectancy of nursing intervention.

\section{REFERENCIAS BIBLIOGRAFICAS}

1. ABDELlaH, F. G. - Criterion measures in nursing. Nurs. Res., New York, 10: 21-6, Winter, 1961.

2. ABDELLAH, F. G. \& LEVINE, E. - What factors affect patients opinions of their nursing care. Hospitals, Chicago, 31: 61-4, Nov. 1957.

3. What patients say about their nursing care? Hospitals, Chicago, 31: 53-7, Dec. 1957.7

4. DICHTER, E. - The patient greatest need is security. Mod. Hosp., Chicago, 83: 56-8, 134, Oct. 1954.

5. HORTA, W. de A. - Enfermagem: teoria, conceitos, princípios e processo. Rev. Esc. Ent. USP, São Paulo, 8 (1): 7-15, 1974.

6. KAMIYAMA, Y. - O doente hospitalizado e sua percepção quanto à prioridade de seus problemas. São Paulo, 1972. (Tese de doutoramento - Escola de Enfermagem da USP). 


\section{ANEXO}

\section{I — IDENTIFICAÇÃO}

Caso $n .^{\circ} \ldots$. . . Hospital

Registro n. ${ }^{\circ}$

Sala......

Nome

Idade ... Sexo

Cor

Estado civil

N. ${ }^{\circ}$ de filhos

Naturalidade

Procedência

Ocupação

Grau de instrução

Religião

Diagnóstico médico

Data de entrada na sala

\section{II - PERgUNTAS}

Data da entrevista Início da entrevista: hora

1. Diga as duas coisas mais importantes que incomodam o $\mathrm{Sr}$. (a) por estar em diálise peritoneal.

$10^{\mathrm{a}}$

$2 .^{\mathrm{a}}-$

2. Diga as duas coisas que fazem mais falta ao Sr.(a) por estar acamado na sala de diálise.

$1 .^{\mathrm{a}}-$

$2 .^{a}$

3. O que o Sr.(a) acha que as enfermeiras devem fazer para o Sr.(a)? Diga duas coisas, mais importantes.

$1 . .^{\mathrm{a}}$

$2 .^{\mathrm{a}}-$

III — OBSERVAÇÕES

Fim da entrevista: hora

Assinatura do entrevistador 